\title{
Introduction to special section on patient-reported outcomes in nonstandard settings
}

\author{
Carolyn E. Schwartz ${ }^{1,2} \cdot$ Dennis A. Revicki ${ }^{3}$
}

Accepted: 11 January 2016/Published online: 23 January 2016

(C) Springer International Publishing Switzerland 2016

Patient-reported outcome (PRO) measures are increasingly being used in unprecedented contexts due to the growth in technology, quantitative methods for developing and evaluating tools, and the accessibility of sophisticated ways of collecting and displaying data. Although many manuscripts that cross our path as editors report on the use of PROs in formal research settings (e.g., randomized clinical trials and epidemiology cohort studies), it is clear that there has been substantial growth recently in the use of social media, smart phone apps, and other computer-based devices. These applications highlight the potential for PROs to be deployed fruitfully in novel settings that can inform and impact population health.

In response to this potential trend, we offer a sampling of 12 articles addressing key topics in applying PROs in nonstandard settings. Selected through a competitive review process, we issued a call for letters of intent for papers related to PROs in nonstandard settings and invited what we thought were the best representatives of this emerging new subfield of QOL research. The resulting 12 articles represent the top $30 \%$ of submitted letters of intent.

This special section can be divided into method-based papers aimed at one of four foci: (1) the added value of modifying the time frame and/or frequency of data

Carolyn E. Schwartz

carolyn.schwartz@deltaquest.org

1 DeltaQuest Foundation, 31 Mitchell Road, Concord, MA 01742, USA

2 Departments of Medicine and Orthopaedic Surgery, Tufts University School of Medicine, Boston, MA, USA

3 Outcomes Research, Evidera, Bethesda, MD, USA collection; (2) the advantages of capitalizing on alternative modes of data collection, such as telehealth, accelerometers, and social media; (3) the evidence base for mixing modes of data collection (i.e., paper and pencil versus computer based); and (4) best approaches for presenting PRO data clinically.

\section{The value of modifying the time frame and/ or frequency of data collection}

Before computer technology became so broadly accessible and so portable, research protocols specified relatively infrequent data collection with a focus on feasibility for the data collection study team, and optimizing participant burden to keep them connected to the study but not overwhelmed. More time points would be a goal for longitudinal observational and/or intervention research, with a 'large' number generally being on the order of four time points. There were of course exceptions, with many data points utilized in time series models, but these tended to use administrative data, and not PRO data.

In the past decade, methods for collecting very frequent PRO data have evolved considerably, enriching study designs without compromising the study budget. Schneider and Stone [1] review ways of incorporating ambulatory and diary methods for assessing self-reported symptoms and well-being, and discuss the key benefits of these methods for PRO research. Carlson et al. [2] present results of a study using 'proximal intensive assessment' (data collected every $4 \mathrm{~h}$ for 7 days) to study psychopathology. Colla et al. [3] present results of a study using weekly data collection in adolescents to study trajectories of subjective wellbeing. 


\section{Capitalizing on alternative modes of data collection}

While the above methods generally rely on internet-based data collection (e.g., smart phone apps), there is a new trend for collecting health data remotely. Schougaard et al. [4] present a 'telehealth' study that enables participants to report their symptoms and health status from home rather than having to present at a clinic. This approach enables more patient-centered care and a more efficient use of healthcare resources. Bennett et al. [5] present the results of a study using a device to count steps (i.e., pedometer) as an adjunct measure of activity in patients. They demonstrate how this passive and continuous assessment can be complement standard PRO assessment in severely ill patients. This approach may also be useful as a proxy in the context of missing data. Marshall et al. [6] compare and contrast symptom cluster patterns in data collected via social media versus a more formal research study. They suggest that social media data may be useful to augment findings from traditional data sources.

\section{Mixing modes of data collection}

In addition to the above question of exploring new methods for triangulating on symptom information, several studies in this special section focus on examining the convergence in paper-and-pencil and computer-based assessment. This question is particularly relevant in an era where people are overloaded with invitations to participate in studies. It has become increasingly difficult to attain what used to be standard response rates of 70-80\% [7], so many researchers seek to combine modes of data collection and let participants choose their preferred mode. While some studies suggest that offering this preference does not actually improve response rate [8], it is nonetheless important to know that the resulting scores are comparable across modes and unbiased. Rutherford et al. [9] present a meta-analysis to determine whether bias is introduced by data collection mode, by use or nonuse of assistance, and by setting (home versus clinic). Sharma et al. [10] compared the psychometric characteristics of paper-and-pencil versus computerized assessment in a clinic setting. Barthel et al. [11] describe the implementation of a computerized-adaptive test as an adjunct to standard pediatric clinical care.

\section{Best approaches for presenting PRO data clinically}

Using PROs in a clinical setting requires adaptations to produce useable and interpretable data in 'real time.' Several articles in this special section examine the effectiveness of various graphical interfaces for different end users. Kuijpers et al. [12] examine preferences for different graphical formats for presenting individualized data in patients and providers, as well as the discrepancy between self-rated and objective understanding of the information. Llewellyn and Skevington [13] evaluated a method for providing guided individualized graphical feedback based on PRO data in a primary care population. Johnston et al. [14] used a graphical display of scores to identify areas of greatest concern for treatment planning on the basis of PROMIS domain scores. All of these studies highlight the use of both qualitative and quantitative data in evaluating graphical interfaces for helping end users interpret PRO scores.

As a group, this set of 12 articles demonstrates novel and useful developments in the field of PRO research. They represent a range of methodological studies and applications of diverse modes, designs, and settings for making the participant's voice central to evaluating health and health care. It is our hope that bringing together this eclectic set of papers will stimulate further creative and rigorous work in this emerging subfield of QOL research.

\section{References}

1. Schneider, S., \& Stone, A. A. (2016). Diary methods can facilitate the measurement of patient reported outcomes. Quality of Life Research (in press).

2. Carlson, E., Field, N. P., Ruzek, J. I., Bryant, R. A., Dalenberg, C. J., Keane, T. M., et al. (2016) Advantages and psychometric validation of proximal, intensive assessments of patient reported outcomes collected in daily life. Quality of Life Research (in press).

3. Colla, L., Fuller-Tyszkiewicz, M., Tomyn, A., Richardson, B., \& Tomyn, J. (2016). Use of ecological momentary assessment data to enhance evaluation of a subjective wellbeing intervention. Quality of Life Research (in press).

4. Schougaard, L. M. V., Larsen, L. P., Jessen, A., Sidenius, P., Dorflinger, L., de Thurah, A., et al. (2016). AmbuFlex: Experiences with large scale implementation of patient-reported outcomes (PRO) in clinical practice. Quality of Life Research (in press).

5. Bennett, A. V., Reeve, B. B., Basch, E., Mitchell, S., Meeneghan, M., Battaglini, C., et al. (2016). Evaluation of pedometry as a patient-centered outcome in patients undergoing hematopoietic cell transplant (HCT): A comparison of pedometry and patientreports of symptoms, health, and quality of life. Quality of Life Research (in press).

6. Marshall, S. A., Yang, C. C., Ping, Q., Zhao, M., Avis, N. A., Ip, E. H. (2016). Comparison of symptom clusters in breast cancer patients derived from social media data and research study data. Quality of Life Research (in press).

7. Dillman, D. A., Smyth, J. D., \& Christian, L. M. (2014). Internet, phone, mail, and mixed-mode surveys: The tailored design method (4th ed.). Hoboken, NJ: Wiley.

8. Ziegenfuss, J. Y., Beebe, T. J., Rey, E., Schleck, C., Locke, G. R., \& Talley, N. J. (2010). Internet option in a mail survey: More harm than good? Letter to the editor. Epidemiology and Infection, 21(4), 585-586. 
9. Rutherford, C., Costa, D. S. J., Mercieca-Bebber, R., Rice, H., Gabb, L., King, M. (2016). Mode of administration does not cause bias in patient-reported outcome results: A meta-analysis. Quality of Life Research (in press).

10. Sharma, P., Dunn, R. L., Wei, J. T., Montie, J. E., Gilbert, S. M. (2016). Evaluation of point-of-care patient-reported outcome assessment in clinic settings: Integration, parallel-forms reliability, and patient acceptability of electronic quality of life measures during clinic visits. Quality of Life Research (in press).

11. Barthel, D., Fischer, K. I., Nolte, S., Otto, C., Meyrose, A. K., Reisinger, S., et al. (2016). Implementation of the Kids-CAT in clinical settings: A newly developed computer-adaptive test to facilitate the assessment of patient-reported outcomes of children and adolescents in clinical practice in Germany. Quality of Life Research (2016).
12. Kuijpers, W., Giesinger, J. M., Zabernigg, A., Young, T., Friend, E., Tomaszewska, I. M., et al. (2016). Patients' and health professionals' understanding of and preferences for graphical presentation styles for individual level EORTC QLQ-C30 scores. Quality of Life Research (in press).

13. Llewellyn, A., \& Skevington, S. (2016). Evaluating a new methodology for providing individualized feedback in healthcare on quality of life and its importance, using the WHOQOL-BREF in the community. Quality of Life Research (in press).

14. Johnston, K., Lawrence, S., Dodds, N., Yu, L., Daley, D. C., Pilkonis, P. A. (2016). Evaluating PROMIS ${ }^{\circledR}$ instruments and methods for patient-centered outcomes research: Patient and provider voices in a substance use treatment setting. Quality of Life Research (in press). 